\title{
SATLABS GROUP: LEADING THE DVB-RCS STANDARD TO A COMMERCIAL SUCCESS
}

\author{
Xavier Lobao \\ European Space Agency, ESA/ESTEC, P.O. Box 299, 2200AG, Noordwijk, The Netherlands
}
Abstract: The paper describes the work of the SatLabs Group in support of the DVB- RCS standard, including a description of the main work areas.

Key words: DVB-RCS; open standard; interoperability; market development.

\section{INTRODUCTION}

The DVB-RCS is an open standard to provide a return channel via satellite to systems based on the DVB (Digital Video Broadcast) standard. The standard was originally defined by the European Space Agency (ESA) and a group of satellite operators and then was refined by an industrial group under the umbrella of the DVB Project. The standard is published by ETSI (European Telecommunications Standards Institute) and maintained by the DVB Project.

An effort was made to accommodate in the DVB-RCS standard a wide range of commercial requirements and to define an efficient and future-proof standard for many application scenarios. As a consequence, the standard is complex and full of options. The standard specifies the air interface and requirements for the user terminal but there is a lot of freedom in the implementation of systems and networks.

ESA has been supporting technically and financially the development of systems, equipments and components based on the DVB-RCS standard since its first definition. 
From this vantage observation point ESA realised the difficulties to ensure interoperability in the presence of so much freedom and choice. Seeing that this might undermine the credibility of DVB-RCS as an open standard ESA decided to take action. As a result of discussions with some of the DVB-RCS stakeholders the decision was taken to create the SatLabs Group to foster interoperability among DVB-RCS products. 


\section{SATLABS GROUP}

The SatLabs Group is an organisation operating under the auspices of ESA but open to the participation of all firms worldwide with a commercial interest in the DVB-RCS standard. Membership is formed at present by some 30 organisations including service providers, satellite operators, system integrators, terminal manufacturers and technology providers. The Group contains a very good representation of all the segments of the value chain and includes the main players in the satcom arena (a list of members is available in www.satlabs.org). The SatLabs Group is a truly global organisation with members from many different areas worldwide.

To date the work of the SatLabs Group is organised in five domains:

- Interoperability

- Low-cost service provision

- Market development support

- DVB-RCS promotion

- Standard evolution

\subsection{Interoperability}

The main objective of the SatLabs Group is to ensure interoperability among DVB-RCS terminals and systems and the availability of solutions for interoperability testing and certification. In line with that objective a great deal of the tasks of the Group have been devoted to verify interoperability and to formalise it into a certification programme.

The Group prepared a test plan with tests aimed at verifying basic compliance with the standard and to ensure interoperability. The Group defined a qualification programme leading to a certification process. In connection with interoperability, SatLabs prepared and published a document containing system recommendations aimed at simplifying interoperability implementation in systems based on the DVB-RCS standard.

For the certification to succeed, the key step is the availability of independent test equipment where to test terminals for compliance and interoperability and an independent test laboratory that carries out testing and certification. ESA is developing the test bed for certification testing for the SatLabs Group. An independent professional testing laboratory will be selected to operate the test bed and execute the qualification programme on behalf of SatLabs. The target is to be operational and start testing and 
certification by the end of 2004. In the meantime, ad-hoc interoperability test campaigns are being organised with interested companies.

The first of such campaigns was carried out during 2003. Interoperability was successfully verified among three manufacturers between all combinations of terminals and systems. This was the proof that DVB-RCS leads to interoperable products and thus the advantages of an open standard can be fully exploited. Another similar campaign is planned for the fall of 2004 involving at least two additional manufacturers.

\subsection{Low cost service provision}

Another of the open issues and main difficulties with the standard, and in general of any satellite communications system, is the high cost of user terminals and in general the cost of DVB-RCS service provision. Given the fact that SatLabs membership includes the main DVB-RCS players, the Group was the natural forum to try to find solutions to reduce the cost of user terminals. The Group agreed to set as an objective the reduction of costs. The main cost drivers were identified: cost of user terminal, of terminal installation and of satellite capacity.

\subsubsection{User terminal cost}

In the emerging phase of a standard, obviously productions volumes are rather low and hence scale economies cannot be fully applied. This leads to high costs, especially of user terminals. The group identified a number of critical components that were the main contributors to the cost of terminals. The Group agreed on common specifications for these critical components. ESA is supporting the development of these components, so that the components can be available openly in the market in larger volumes at lower prices.

The components for which developments are currently under way are:

- Ku- and Ka-band solid state power amplifiers

- Ka-band power line-up, including the power amplifier, drivers and mixers

- DVB-RCS MAC and physical layer chipset

\subsubsection{Terminal installation cost}

Once the cost of user terminals is optimised, another of the main cost drivers is that of user terminal installation. Following the recommendations 
of SatLabs, ESA is running a study to analyse the problem and come up with improvements on architectures, tools and procedures using DVB-RCS that allow a simpler and faster installation process involving less qualified personnel than normally required in interactive satellite installations.

The goal is to optimise the installation process, using the DVB-RCS to reduce the need for intervention of network operations personnel, to minimise the need for specific instrumentation by installers and to simplify antenna pointing and terminal configuration. Ideally, the installation would be carried out by end users or by satellite TV installers without the need for specialised skills.

\subsubsection{Satellite capacity cost}

The cost of satellite capacity is the dominating operating cost in any satellite communications system. In the long term, optimised satellite systems for broadband access are expected to be launched, using Ka-band and multiple narrow spots. However, in the mean time services are provided using existing satellite capacity in $\mathrm{Ku}$-band satellite systems with broad beams.

Work on reducing the cost of satellite capacity has in recent times been carried out outside of the SatLabs Group by standardisation groups, mainly with the definition of DVB-S2 with adaptive coding and modulation, which increases significantly the efficiency of the satellite link. The SatLabs Group is prepared to issue recommendations on optimised target satellite architectures, equipment and procedures to most efficiently use the satellite bandwidth.

\subsection{Other work areas}

The SatLabs Group is also working on simplifying the development in the market of systems based on the DVB-RCS standard. This involves areas like application support, integration with operational support systems and support for the efficent provision of emerging services, such as voice-overIP.

A significant example of the kind of tasks that SatLabs is carrying out is in the area of PEP (Protocol Enhancing Proxies). In a satellite communications system, PEP solutions are required to ensure a good level of end-user performance for applications based on the TCP and HTTP 
protocols. Products exist in the market but the lack of interoperability between them forces DVB-RCS system and terminal manufacturers to select a particular solution or to spend large efforts to integrate new PEP products into their terminals and systems. The Group is defining a standardised interoperable PEP that will guarantee a good level of performance for TCP and HTTP applications whilst allowing different PEP products to be used in the terminals and the gateways and ensuring full interoperability with futureproof solutions.

Recognising that for a new standard to be successful in the marketplace it is important that information is available for potential users, operators and developers, the SatLabs Group decided to become a knowledge centre for DVB-RCS. Application notes, descriptions of pilot experiences, guidelines and recommendations are being published to raise awareness of the capabilities of the DVB-RCS standard and to simplify the set-up of applications and services.

Last but not least, the Group is continuously analysing possible improvements of the standard so that in the regular maintenance opportunities that are likely to occur the improvements are incorporated into the standard.

The SatLabs Group has an open work programme in support of the DVBRCS standard, with new tasks being defined by a committed and proactive membership. 


\section{CONCLUSIONS}

In conclusion, the SatLabs Group is being crucial in the commercial success of the DVB-RCS standard. The verification and formalisation of interoperability provides the proof that gives credibility to an open standard. The Group is providing the elements that will allow affordable implementations that satisfy user needs in terms of applications, functionality and performance. By providing relevant information in the public domain the SatLabs Group is enabling the DVB-RCS community to grow with the incorporation of innovative, low-cost solutions and to simplify the integration of applications and the realisation of demanded services.

More detailed information about the SatLabs Group, its membership, the work programme and public domain information published by the SatLabs Group can be found in the SatLabs web site, www.satlabs.org. 